\title{
Understanding the Process of Generalization in Mathematics through Activity Theory
}

\author{
Gabriela Dumitrascu \\ Eastern Michigan University \\ Ypsilanti, USA
}

\begin{abstract}
The practice of generalization is a powerful process that should be present in mathematical learning from kindergarten to college. It is crucial for teachers at all school levels to have a deep understanding of the process down to knowing its genetic decomposition. Activity theory framework provides basic principles that allows us to define generalization as an activity that is socially and historically developed through tools and artifacts mediations, internalization of social knowledge, and that is transformed through learning and development I present the means of the generalization activity using Leontiev's activity theory intertwined with Rubinshtein's description of the generalization process. This theoretical framework may also support teacher educators and teachers while they use highleverage teaching practices such as: eliciting and interpreting individual student's thinking, diagnosing particular common patterns of student thinking and development, or leading a group discussion.
\end{abstract}

Keywords: Activity Theory; Davydov; Vygotsky; Leontiev; generalization.

\section{Introduction}

In mathematics, we are well used with statements as the following ones:

1. Find the general term for the sequence: $1,5,9,13,17, \ldots$

2. Find a formula for computing the measure of the interior angle of a regular polygon with $\mathrm{n}$ sides.

3. Consider the sequence defined recursively by

Find an explicit formula for the $n$th term.

$$
\begin{aligned}
& x_{1}=\sqrt{2} \\
& x_{n}=\sqrt{2}+x_{n 1}
\end{aligned}
$$

4. The sum of any two consecutive integers is odd.

5. The segment connecting the midpoints of two sides of a triangle is parallel to the third side and is half as long.

6. The first derivative of an increasing function is positive. 
From a mathematician's perspective, these statements request mathematical computations; from a psychologist's perspective, the statements demand a higher level of thinking; for mathematics educator's perspective, these statements are learning outcomes of a complex process. All three perspectives have a common target: the process of generalization.

As mathematics teacher, I see the above statements divided into two categories of generalization. The first category of problems $(1,2$, and 3$)$ asks to search for a general statement and the second category $(4,5$, and 6$)$ asks to search for a generalization process. We use to teach and to learn these categories as represent different mathematical entities. However, they are parts of the same process: the process of generalization.

As mathematicians, we are so used to focus on content and reasoning development when we learn and teach mathematics, and we seem to forget that generalization is the "heartbeat" of mathematics (Mason, 1996):

"Generality is so central to all of mathematics that many professionals no longer notice its presence in what is, for them, elementary. But, it is precisely the shifts of attention that experts have integrated into their thinking, which are problematic for novices." (Mason, 1996)

Mason stresses that mathematicians, teachers, and even mathematics educators are too used to perform some thinking processes and they become unaware of them. Consequently, we need to become aware that the process of generalization is one of the most powerful thinking processes, and to understand its decomposition when we examine a mathematical situation.

\section{From Literature Review}

In the mathematics literature, generalization can be seen as a statement that is true for a whole category of objects; it can be understood as the process through which we obtain a general statement; or it can be the way to transfer knowledge from one setting to a different one. Most of the studies that looked at the place of generalization in mathematics instruction choose to analyze the introduction of algebra to young students. There are studies that researched how to create activities for "awakening of pupil sensitivity to the nature of mathematical generalization and dually, to specialization" in order to improve students' algebraic thinking. In other words, how to get students comfortable to see " $a$ generality through the particular" and "the particular in the general" (Mason, 1996). Lee (1996) considers that functions, modeling, and problem solving are all types of generalization and proposes that algebra should be taught through patterns.

Another set of studies on generalization is researching ways to develop algebraic thinking at the elementary school children. J. J. Kaput, D. W. Carraher, and M. L. Blanton (2008) brought a comprehensive collection of research studies together that investigated the introduction of early algebra in elementary school in which generalization plays a crucial role. Note that the contributors to that volume consider "early algebra" a context to develop the potential for generalization at 
elementary schoolchildren and not the introduction of algebra concepts in the early school years. This is a crucial difference highlighted by Kaput. Early algebra focuses on making elementary students comfortable with the process of generalization, which enhances algebraic thinking.

From outside of the mathematics education field, many educational psychologists researched how the thinking process manifests through the process of generalization. Vygotsky (1986) considered that every concept is the result of a process of generalization. He emphasized the distinction between spontaneous/everyday concept, where the generalization means to move from particular to general, and scientific concept, which means to see the particular from the general. Piaget (1964) considered two different experiences that promote learning: 1) the physical experience that consists in manipulation of objects and in drawing some knowledge by abstractions from the objects; 2) the logical-mathematical experience in which knowledge is drawn by abstracting from the actions effectuated upon the objects. Rubinshtein (Davydov, 1990) made the difference between two types of generalization: the empirical generalization that is the result of comparing and identifying the external characteristics that are similar or identical to things; and the theoretical generalization that is the result of analysis and abstraction that happen while the data received through senses is transformed in order to determine the essence of things. Krutetskii (1976) identified two ways in which schoolchildren learn mathematical concepts through generalization. The first method is the empirical generalization, which consists in a gradual generalization by analyzing a sequence of concrete examples in which the nonessential attributes are systematically changed. This method is used by the children that are not or almost not successful in mathematics learning to master the general mathematical knowledge. The most successful children in learning mathematics are using a different way to approach the generalization. They are able to generalize a solution, which is unique to a theoretical generalization, just from a single example by identifying the internal connections/relationships involved in the solution.

In the book, Types of Generalization in Instruction: Logical and Psychological Problems in the Structuring of School Curricula (1990), V. V. Davydov discussed his concern that the empirical generalization used in traditional Russian instruction is one of the sources that create difficulties for schoolchildren to master the instructional material. Regarding the case of mathematics, Davydov used the findings of Krutetskii's research on mathematical abilities to propose a generalization-based instruction at the elementary school levels. Several experimental studies conducted in the Unites States (Dougherty \& Slovin, 2004; Moxhay, 2008; Schmittau, 2003; Schmittau, 2004) based on Davydov's instructional theory showed that the participating children were able to perform theoretical reasoning based on generalization by the end of the second or third year of instruction.

Analyzing the studies that were done so far from the lens of a teacher that looks to find ways to help students finalize a generalization process, I noticed that: the term "generalization" is heavily loaded in mathematics. Even if we consider that 
it is most commonly understood as a duality between going from particular to general and seeing the particular through the general, this interpretation does not include a description for the reasoning involved in the process of generalization that would allow teachers and educators to design a generalization-based instruction.

In my quest to understand the needs of pupils to achieve higher-level thinking, which implies generalization, I found the theories developed by the educational psychologists A. N. Leont'ev (1903-1979), Krutetskii (1917-1991), S. L. Rubinshtein (1889-1960), and Dubinsky to provide a supportive background. From their perspectives, I will interpret the process of generalization in mathematics as follow:

1) An activity system that contains specific actions and operations (in the way Leont'ev [1978] defined the concepts of activity, action, and operation);

2) It has two specific motives:

a. to identify something general that is already known in particular cases and to apply the general to the particular, and

b. to find something general that is not known from isolated and particular cases (as Krutetskii [1976] discovered in his research on schoolchildren mathematical abilities);

3) It has three main ways to be performed: empirical generalization, pseudo-generalization, and reflective generalization (which are the three "routes" to generalization described by Rubinshtein [Davydov, 1990], where I incorporate the definitions of empirical abstraction, pseudoabstraction, and reflective abstraction given by Dubinsky [1991]).

Next, I will discuss these theories as a framework, which may provide foundation for developing the pedagogical content knowledge needed to construct settings for students to participate in a activity of generalization.

\section{Theoretical Framework}

Vygotsky considered that all the concepts learned by humans become internalized through a process of generalization. He classified the internalized concepts into spontaneous/everyday concepts and scientific concepts. The spontaneous/everyday concepts are created by child's personal experience, and they can form without systematic instruction. The scientific concepts are what the child cannot directly observe or experience. They should be taught to the child by creating conditions in which the child studies the formation of an "artificially devised experimental concept" (Vygotsky, 1986, p. 161). The two types of concepts form a unitary process; they are continuously related, and reciprocally contribute to each other's development. The overall development of a child and learning are two simultaneous processes that depend qualitatively on each other (Vygotsky, 1986). The qualitatively duality between development and learning is essentially based on the types of experiences that the child has. The everyday experience of a child provides knowledge from a direct physical contact with the environment when the child uses his/her senses to analyze, compare, classify, 
and synthesize. Through a systematic instruction, the child experiences "artificially designed" situations where in collaboration with another more knowledgeable peer or an adult he/she analyzes, compares, classifies, and synthesizes at a high psychological level and the child travels his/her Zone of Proximal Development (ZPD) (Vygotsky, 1978).

Besides the strong opinion that Piaget had against Vygotsky's theory about the duality of the development and learning (Piaget, 1964), he also identified, similar to Vygotsky, two different experiences that a child should go through during a learning process: physical experiences and logical-mathematical experiences. First, there are physical experiences that consist on having direct contact with objects. During this experience, knowledge is drawn from the physical properties of the object by making abstraction from them. Second, there are logical-mathematical experiences, in which knowledge is drawn through actions effectuated on objects. The objects are physically present, but there is also the set of actions that modify the objects, which transforms the process into a learning experience.

Vygotsky's and Piaget's perspectives on learning are complementary to each other in the following way: Every day concepts are predominantly learned through physical experiences, where scientific concepts are predominantly learned through logical-mathematical experiences. Rubinshtein's theory of thought and Krutetskii's work on schoolchildren abilities also support this combined theory of learning.

Rubinshtein divided human thoughts into empirical/visual thoughts and theoretical/abstract thoughts. The empirical thought is the result of comparing and identifying the external characteristics that are similar or identical in things. The theoretical thought is the result of analysis and abstraction that arise while the data received through senses is transformed in order to determine the essence of things.

Krutetskii, through his research on schoolchildren mathematical abilities, was able to provide concrete examples of children thinking that reflect the two categories of thoughts described by Rubinshtein. Krutetskii (1976) discovered two ways in which schoolchildren learn mathematical concepts through generalization. The first method, named empirical generalization, consists in a gradual generalization by analyzing a series of concrete examples in which the nonessential attributes are systematically changed. The children, who are not or almost not successful in mathematics learning, use this method in order to understand general mathematical knowledge. The most successful children in learning mathematics are using a different way to approach the process of generalization. They do a theoretical generalization. They are able to generalize a solution just from a single example by identifying the internal connections/relationships involved in the task. These children are generalizing solutions and methods to approach a problem instead of generalizing particular or external aspects of a problem. 
The experiment conducted by Krutetskii and his collaborators was quite sophisticated and laborious. He selected schoolchildren from $6^{\text {th }}$ and $7^{\text {th }}$ grade that did not receive any instruction in algebra. An experimenter taught a child the way in which the square of a binomial may be obtained through "the formula for short multiplication", that is: $(a+b)^{2}=a^{2}+2 a b+b^{2}$. Then, the child was asked to solve a sequence of tasks similar with the following:

1. $(a+b)^{2}=$

2. $\left(1+a^{3} b^{2}\right)^{2}=$

3. $\left(5 x+0.6 x y^{2}\right)^{2}=$

4. $(3 x \quad 6 y)^{2}=$

5. $(m+x+b)^{2}=$

6. $\left(4 x+y^{3} a\right)^{2}=$

7. $51^{2}=$

8. $(C+D+E)(E+C+D)=$

The order of these examples depended on their level of generalization. After the child who was interviewed became familiar with the short multiplication formula for the square of a binomial, the experimenter asked the child to solve task 8 . If the child was not able to solve it correctly, the experimenter proposed task 2 and, if it was necessary, helped the child to become comfortable solving these types of problems. Then, again, the experimenter asked the child to solve task 8 . If the child was not successful, the experimenter showed task 3 . The procedure was repeated until the child solved correctly the task 8 . Krutetskii analyzed the number of iterations needed for each child. The findings of this analysis revealed that the children who used a theoretical generalization, at some point during the interview, were able to solve example 8 faster than the ones who based their method of solving the problems only on empirical generalization.

For example, one child was able apply correctly the short formula for the square of a binomial right after experimenter's presentation. Therefore, through interaction with the experimenter, the child learned a scientific concept: $(a+b)^{2}=a^{2}+2 a b+b^{2}$. Next, a new situation is presented to the child, the product $(C+D+E)(E+C+D)$. First, the child analyzed the external characteristics of this example and from this physical experience he learned that it is not a binomial, and it is not a square. He had a strategy in mind for solving the problem, but it was a laborious one. He said: "But that will be 9 terms. That's a lot." This triggered him to transform the row data. The child started now to experience a logical-mathematical process. He commuted the first term $E$ from the second parenthesis from the first place to the last one and obtained: $(C+D+E)(C+D+E)=(C+D+E)^{2}$, the child transformed the information given. Next, he grouped the last two terms together and obtained: 
$[C+(D+E)]^{2}$. At this moment, the child ignored the physical characteristic of $(D+E)$ therefore he performed an abstraction. It was essential for the child that $(D+E)$ had the role of the second term in the expression $[C+(D+E)]^{2}$. From this point, all the conditions needed to use the short multiplication formula for the square of a binomial were satisfied, and the child solved the example correctly.

The thinking process of the child from previous example is a clear representation of the complementarity of Vygotsky's and Piaget's views about the learning process. At the beginning, the child in his/her interaction with an adult learned a scientific concept: the short multiplication formula for the square of a binomial. Then the child came to learn a new concept, a spontaneous one, which was the short formula for the square of a trinomial by physical contact with a new example and by logical/mathematical transformations of this example.

Things happened different with another child. In this case, the child became comfortable to solve problems of the form $(a+b)^{2}$ and was able to solve correctly the example $(2 x+y)^{2}=4 x^{2}+2 \times 2 x \times y+y^{2}=4 x^{2}+4 x y+y^{2}$. But when the child saw the example $1+\frac{1}{2} a^{3} b^{2} \div$, he/she said that this is different because $a$ and $b$ are not separated by ' + '. The child wrote $\frac{1}{4} a^{6}+2 \frac{1}{2} a^{3} b^{2}+b^{4}$, which shows that the child was able to manipulate physically the parts of a squared binomial only if they are externally identical (in this case each term had to contain a letter) with the parts of the given formula.

The previous example shows that the child was not able to overcome the physical experience and enter in the realm of the logical-mathematical experience by himself. Therefore, from Piaget's perspective the child did not complete the learning process since he was not able to generalize the short multiplication formula for the square of the binomial. From Vygotsky's perspective, the child should develop his Zone of Proximal Development by interaction with an adult in order to learn the scientific concept of the short multiplication formula for the square of the binomial.

A conclusion that Krutetskii drew from his experiment was that the mental actions, used by the successful children in order to make generalizations, are qualitatively higher than the ones used by the children that are not successful in math. The successful children are performing theoretical generalizations while the less successful children are getting stuck in empirical generalizations. The ability to do theoretical generalizations helps the schoolchildren, from the first group, to solve problems that are different in context and to overcome situations that are new to them. The absence of this ability makes the children to be afraid to tackle anything that is not familiar to them.

In the book Types of Generalization in Instruction: Logical and Psychological Problems in the Structuring of School Curricula (1990), V. V. Davydov discusses his 
concern that the empirical type of generalization used in traditional Russian instruction is one of the sources that create difficulties for schoolchildren to understand the instructional material. Regarding the domain of mathematics, Davydov is using the findings of Krutetskii's research on mathematical abilities to examine the effect of Russian traditional instruction on schoolchildren mathematical thinking. He analyzed how the concept of number is developed in traditional Russian instruction. He looked at Russian's first grade textbooks, at the methods books used to prepare teachers for elementary school, and interviewed first-grade students. Davydov found that many of the $1{ }^{\text {st }}$ grade students were not able to comprehend the unit as a relationship between the physical parts of an object and the action of measurement. Davydov wrote:

"Solving the essential problems in contemporary school education is ultimately linked to changing the type of thinking that is projected by the goals, content, and methods of instruction. The entire instruction system must be reoriented from the children's development of rational-empirical thought to their development of modern theoretical scientific thought." (Davydov, 1990)

With his colleague El'konin, Davydov designed a content-based generalization curriculum for the elementary school. Their approach of instruction was considered at that time, and it still continues to be regarded today, as 'quite' exotic because:

“The 'technique' of forming content-based generalization is quite different from the one that is peculiar to empirical generalizations. A transforming, object-related action and an analysis that establish essential connections in an integral entity, its genetically original (universal) form, rather than observation and comparison of the external properties of objects (traditional visuality), serve as the basis for this process. Here, discovery and mastery of the abstract and universal precedes mastery of the concrete and particular, and the concept itself as a certain method of activity serves as a means of ascending from the abstract to concrete." (Davydov, 1990)

The mathematics curriculum designed by Davydov was oriented toward children's formation of content-based abstractions and the development of theoretical thought. The goal of the curriculum was to create a theoretical understanding of the real number, which is the concept of quantity. First grade students begin their experience by measuring, comparing different quantities by singling out their basic properties. They analyze the relationships that exist between and within quantities, and they start to record them using symbols, which are letters for quantities and signs $(e . g .,=,<,>)$ for relationships between quantities. Next, the children analyze the changes that may occur in a quantity and they indicate the change by using the signs ' + ' or " - ". Only after these experiences, the children are introduced to the number concept as a way of representing a relationship among quantities:

$\mathrm{A} / \mathrm{C}=\mathrm{N}$, where $\mathrm{N}$ is any number, $\mathrm{A}$ is any object represented as a 
quantity, and C is any measure. (Davydov, 2008)

This definition implies that any number is the result of a measurement. If we have an object and we see the object just through one of its measurable features (e.g., a segment is represented by its length) and we have a measure (e.g., a paper clip), then the result of measurement is a number (e.g., the result of measuring the length of the segment with the paper clip is a number).

The following example shows how the concept of whole numbers multiplication may be introduced by using content-based generalization curriculum. A teacher brings in front of the children a large and heavy bucket filled with water a very small cup. He asks them to find out to how many rabbits they can give water if each rabbit would get one small cup of water. The students give the answer right away: they need to measure the water from the bucket with the small cup. A child starts measuring and counting the glasses. The work is laborious, and it will last a long time to be done. In the process, some of the water spilled over so the teacher points out that the measuring will not be accurate. But children continue the work, and are happy with their solution, even if it would take the whole class to finish the job, and even if the number found would not represent exactly the number of rabbits. Teacher's responsibility is to reorient the children from having only physical experience to start having a logical-mathematical experience. He points out how inefficient is the procedure performed by the kids because of the little unit that they are using to measure the water. However, the problem has to be solved. Therefore, the students have to "think" to find another method that will provide an answer faster and more accurate. After several trials, the teacher suggests setting aside this problem, and trying to solve another situation. This time, the children have to find out from how many bricks were used to build the wall shown in a picture that hangs in front of the classroom. The side of the wall from the picture is $52 \mathrm{~cm} \times 75 \mathrm{~cm}$, and it is made out of bricks represented by rectangles of the dimensions $2 \mathrm{~cm} \times 3 \mathrm{~cm}$. Children start to count the bricks from their sits straggling to keep the counting strait. Teacher notices how hard it is to count the bricks from faraway, so he calls a student to come closer and count the bricks. The whole class follows the student's counting. They would have continued counting because in this way they would find the number of bricks in the wall, but the teacher stops them and makes them understand how long time they have to spend counting even if they are extremely good counters. This is another situation, beside the first one where they had to count the little glass of water, in which the method of counting takes too much time to find the answer, and many mistakes can be made during the process.

The teacher's intention is to motivate the children to look for another method to solve the problem. He wants to make his students look for a method that requests more than just physical experiences. The teacher has to guide his students through a setting that may contribute to the development of their ability to have a logical-mathematical experience. First, the teacher does not let the students find the answer by counting and points out how the counting method is time consuming and inefficient. Second, he asks children to "think," 
to search for another method, even if they are not able to provide satisfactory answers. However, the setting is created for children to reach their Zone of Proximal Development and the teacher leads them to the most expected logicalmathematical experience.

In the next part of the lesson, the teacher asks children to do a series of actions that transform the process of counting with the little cup. He shows them that if they use a bigger unit of measure, a mug, it is easier to measure the water from the bucket. Then he leads children to discover the relationship between the mug and the little cup. At the end, children are able to find by themselves the total number of the little cups that filed the bucket by using the new method.

If we go back at the beginning of the lesson and look for its outline, we can clearly identify the theoretical background. First, the teacher created the setting that position the students in their ZPD (Vygotsky), he makes sure that students have physical and logical-mathematical experiences (Piaget), and he focuses on having his students generalize methods of solving (Krutetskii).

I started this section by presenting some theoretical perspectives on learning and at the end I presented an example of a lesson created on the foundation of these theories. This section was an example of how a theoretical framework can inform and transform teaching practices. In the next part, I will take a similar approach and I will present a way to think about generalization in mathematics that may be a theoretical framework to support teacher educators and teachers while they research and use high-leverage teaching practices such as eliciting and interpreting individual student's thinking, diagnosing particular common patterns of student thinking and development, or leading a group discussion.

\section{A Definition for Generalization in Mathematics}

The concept of generalization is most commonly understood as a duality between going from particular to general and seeing the particular through the general. In order to provide an understanding of the thinking involved in a mathematical generalization process, I describe in this section the process of generalization from the perspectives of the theories developed by the educational psychologists A. N. Leont'ev (1903-1979), Krutetskii (1917-1991), and S. L. Rubinshtein (1889-1960), and of contemporary mathematics educator Ed Dubinsky.

\section{The Human Activity in Leontief's Interpretation}

Leont'ev (1978) defined human activity as "a process in which mutual transfers between two poles "subject-object" are accomplished" (p. 50). Also, Leont'ev stressed that an activity has to be understood as "a system that has structure, its own internal transitions and transformations, its own development" (p. 50). An activity is not a reaction or a set of reactions to different conditions. For an activity to occur, it has to be a need for something. When the need is "disclosed" it becomes a motive for the activity (p. 116). In other words, when the decision to satisfy a need is taken, the need becomes a motive for an activity. From this perspective, an activity and its motive are "necessarily" connected (p. 62). 
Furthermore, a need is the motive that triggers an activity. The goal of the activity is to satisfy the need. Most of the times, the goal of an activity cannot be attained through a single process. Therefore, it has to be divided into a series of sub-goals and a sequence of goal-oriented processes has to be constructed. Each of these processes is an action subordinated to a particular purpose. At its turn, each action is performed through a set of rules and laws (named operations) that had been historically established. These operations are applied to the particular conditions imposed by the purpose of the action. In conclusion, the transfer between the two poles, subject and object, generates the interaction between the subject, represented by the subsystem \{need, motive, goal, purpose, conditions\}, and the object, represented by the subsystem \{activity, actions, operations\}. Harry (2008, p.119) used the following graphical representation in order to capture the characteristics of an activity with their interactions.

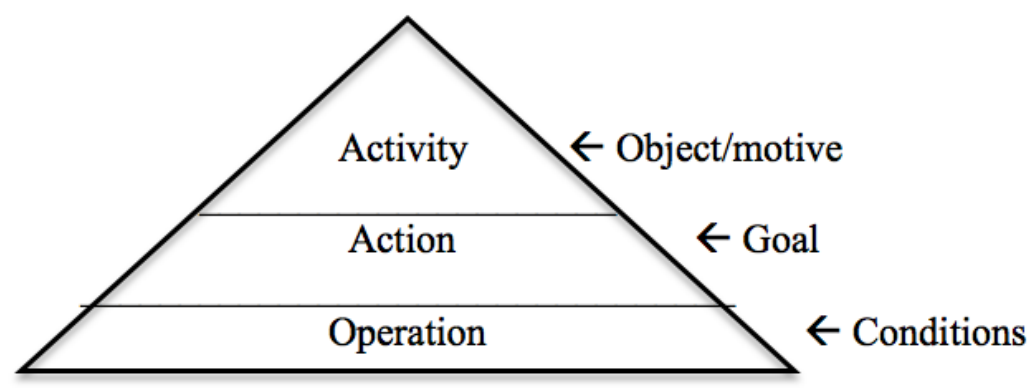

Figure 1: The schematic representation of an activity, Harry (2008, p. 119).

I will consider a similar schematic representation of an activity, which is given in Figure 2. In this schemata, the interaction between "subject" and "object" is represented through arrows that should be read in the following way:

- a need triggers a motive;

- the motive triggers an activity;

- the activity is associated with a genetic goal that is subordinated to the need;

- the generic goal triggers an action or a chain of actions;

- each action is associated with a purpose that is subordinated to the generic goal;

- each action consists in performing a set of operations. 


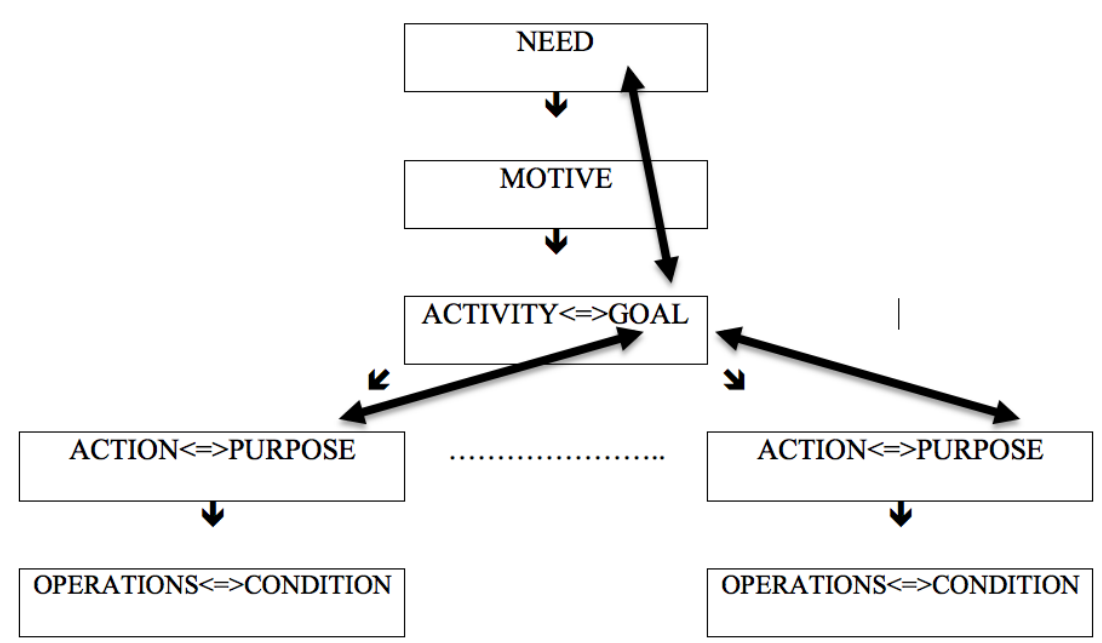

Figure 2: The schematic representation of a system that is characteristic to an activity

Let us expand of Leontief's example of learning to drive a car (Leontief, 1978, p. 66). I live in a house located at the position A, and my work place is located at the position B. Each weekday, I have to go from my house to my work place. Therefore, I need to commute from A to B. This need that I have identified has to be satisfied through an activity that I should perform. There are several activities that I can perform to move myself from point A to point B, e.g., walking, biking, driving a car, or using public transportation. I choose the option of driving a car. Consequently, in order to satisfy the need to commute from A to B, I choose to perform the activity of driving a car for the motive (the need is transformed into a motive) to commute from my house to my work place. My goal is now to drive a car. To be able to drive a car, I have to perform a chain of actions: for the purpose to make the car to move, I have to start the engine of the car; for the purpose to get car on the road, I have to orient the car to move in a certain direction; for the purpose to make the car stop, I have to stop the engine of the car. Each of the actions that I already enumerated is accomplished through a set of operations, which are already well-established methods. The procedure for starting the engine of my car consists on pushing the START button while keeping the brake pedal down. To make the car move in the direction that I want, I have to position the automatic shift, accelerate by pushing the acceleration pedal down, and rotate the steering wheel. To stop the car, I have to push down the brake pedal until the car stops moving, bring the automatic shift in the P position, and press STOP button. The operations that I just enumerated are particular for the Nissan Altima 2008 car that I drive. For a different car, there may be different operations involved. Also, the actions that I mentioned above are triggered by the activity of driving a car. If I had chosen the activity of biking with the goal to ride a bike, I would have had different actions and different operations. Therefore, once chosen the activity that has the goal to satisfy the need, the actions are purposefully performed to achieve the goal of the activity.

In conclusion, an activity, from Leontief's perspective, has to have the following identifiable components:

- the need that motivates the activity; 
- the goal of the activity and how it would satisfy the need;

- the sub-goals that would lead to achieve the goal of the activity;

- the actions that need to be performed and how their purposes would meet each sub-goal;

- the operations involved in each action and how they apply and transform in the particular conditions of the activity.

At this point, it is important to mention that since Leontief activity theory continued to develop and to expand its interpretations and implications. Engestrom (2001) talks about three generations of research that contribute to the evolution of the activity theory. The first generation is centered around Vygotsky's work on the triad of human development Subject-Object-Mediation. The second generation is established by Leontief's differentiation between individual action and collective activity. And, the third generation introduces the interconnectivity and interaction of at least two activity systems. All the contemporary research on activity theory (Jooganah \&Williams, (2016); Gedera \& Williams (2016); Solomon, Croft, Duah, and Lawson, 2014) is part of the third generation. The approach that I am taking, goes back to the second generation and I am considering the process of generalization in mathematics as being the individual activity system that has the characteristics enumerated in the previous paragraph.

\section{Mathematical Generalization as Activity System}

In mathematics, the need for solving a mathematical situation becomes the motive to identify an activity that has as goal to discover a solution accepted by the mathematical community. This activity is, in fact, a mental activity or a mental process. As a mental activity it is generated and determined by mental actions such as analysis, synthesis, and abstraction identified by Rubinshtein (1994) as critical components in a process of thinking. Each of these actions has the purpose to contribute to the process of solving the mathematical situation. All these actions, in order to be performed, request a number of operations that need to be manipulated in the specific conditions imposed by the problem that has to be solved.

The requirements of an activity system were enumerated in the previous section. In this section, I will show how the process of generalization satisfies all these requirements. First, I will discuss the goals of generalization and how they correspond to the need of solving a mathematical problem. The arguments that I will use here are based on Krutetskii's work on students' mathematical abilities, which provides evidence of how children do generalization in mathematics. Next, I will describe the key actions involved in the process of generalization, which are analysis, synthesis and abstracting. Then, I will describe the interrelationships that exist in a generalization activity by using Rubinshtein's "routes" through generalization. In other words, the next part of this section will deplete the schemata from Figure 3, which is a transformation of the activity schemata represented in Figure 2. 


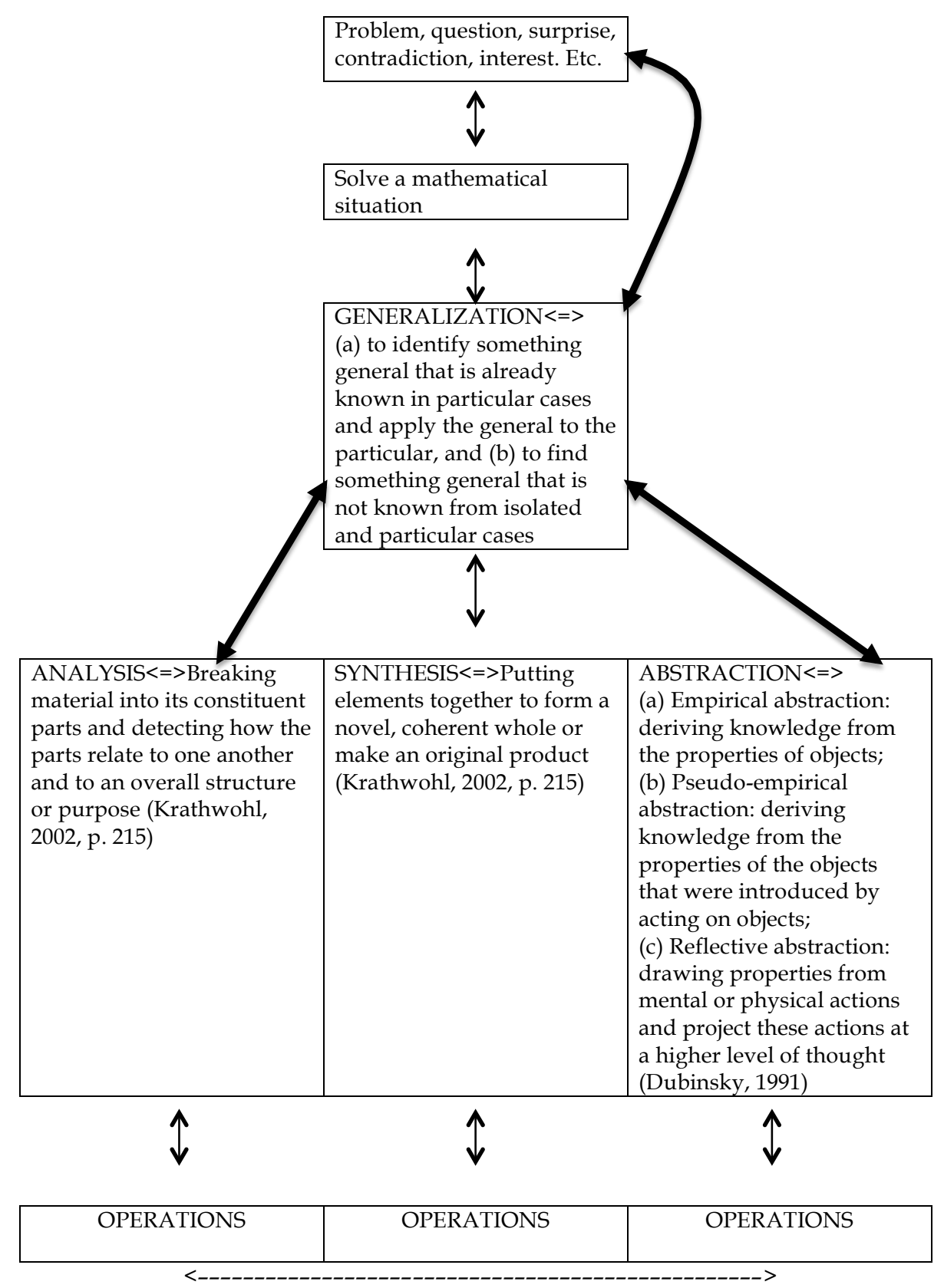

Figure 3: The schematic representation of the system that is characteristic to the process of generalization in mathematics seen as an activity.

\section{The Goals of the Generalization Activity}

To start, I want to highlight one more time that generalization is considered a process and not a result (Rubinshtein, 1994, Krutetskii, 1976). Rubinshtein (1994) proposed to study thinking as a process (a generalization process) that derives from mental activities such as analysis and synthesis instead of studying the assimilation of knowledge, which is a result of a thinking process. Krutetskii (1976) followed Rubinstein's approach and investigated what abilities are needed in order to learn mathematics. The meaning for abilities that Krutetskii 
used in his research is that of "individual traits of mental activity on which the relative swiftness of mastery of skills and habits and their qualitative distinctions depend" (p. 14). Also, Krutetskii noticed that: "the aptitude for learning mathematics is manifested by a pupil's ability to generalize mathematical material" (p. 24).

Generalization is a mental process that supports mathematical learning. Krutetskii (1976) differentiated between two levels of the ability to generalize mathematical materials:

(1) the ability of a child to identify something general that is already known to him in particular cases and apply the general to the particular, for example, to apply the distributive property of multiplication over addition to find the product 618 ;

(2) the ability of a child to find something general that is not known to him from isolated and particular cases, for example, to find the 100 term for the sequence: $1,5,9,13,17 \ldots$. Consequently, the goals for the activity of generalization are:

(1) to apply a general concept to a particular situation;

(2) to discover a general concept from particular cases.

The actions of analysis, synthesis, and abstraction

Rubinshtein (1994) considered that mental actions such as analysis, synthesis, and abstraction have to take place in order to perform a generalization activity. Davydov (1990) describes these actions in the following way:

- Analysis is the method or logical technique by which the objects are represented by observed common attributes (p. 44). This action has the purpose to identify the characteristics that some given objects have in common. It is performed through operations that lead to know each object. The operations used delineate how an object is similar, or identical with other objects. These common properties are called the attributes of the object (Davydov, 1990, p. 38).

- Synthesis is the method or logical technique that uses the attributes observed through analysis to create a new system (p. 44).

- Abstraction is the mental delineation of certain properties of objects and the segregation of them from all other properties (p. 38).

Krathwohl (2002) gives similar descriptions for analysis, synthesis in A revision of Bloom's Taxonomy: An Overview. Dubinsky (1991) discusses the process of abstraction in Reflective Abstraction in Advanced Mathematical Thinking. For a more detailed presentation of the generalization activity, I will further describe the meanings that analysis and synthesis have in the Revised Bloom's taxonomy and how Dubinsky interpreted Piaget's three types of abstraction in the study of mathematical thinking.

The process of analysis implies to break the "material into its constituent parts and detecting how the parts relate to one another and to an overall structure or purpose" (Krathwohl, 2002, p. 215). Its subcategories are differentiating, 
organizing, and attributing. Assume, for example, that we have the following activity: Use the distributive property of multiplication over addition to find the product 7 19. The process of analysis starts by differentiating the parts of the activity: "use the distributive property of multiplication over addition" and "find the product 7 19." First, I focus on the first part, which refers to the general property $a(b+c)=a \quad b+a \quad c$ or $(b+c) \quad a=b \quad a+c \quad a$. Then, I consider the second part, which refers to the product of two whole numbers 7 and 19. The number 7 is one digit. The number 19 is a two digit number. The general property involves two operations, multiplication and addition, e.g., $a(b+c)$ and $a b+a c)$. The second part contains only a multiplication, $7 \times 19$. For the purpose of finding the product 719 using the distributive property of multiplication over addition, the expression has to contain an addition attached to the multiplication. I know that any of the numbers 7 or 19 can be written like a sum, e.g., $7=3+4$ and $19=10+9$. At this point, I finished analyzing the data provided. Next, I have to perform the process of synthesis.

As another example, consider the following activity: "what is the $100^{\text {th }}$ term in the sequence: $1,5,9,13,17 \ldots$ ?" The parts of this activity are: "what is the $100^{\text {th }}$ term" and the "sequence: $1,5,9,13,17 \ldots$. " I will focus on the second part, the sequence. Its first 5 terms are, 1, 5, 9, and 17, which are all odd numbers. However, they are not consecutive odd numbers. It is 1 , skip 3, 5, skip 7, 9, skip 11,13 , skip 15, 17. Also, I see that 5 is with 4 more than $1 ; 9$ is with 4 more than 5 ; 13 is with 4 more than 9; and 17 is with 4 more than 13 . At this point, the process of analysis ends. The next action is the process of synthesis.

Synthesis is the process that brings "elements together to form a novel, coherent whole or make an original product" (Krathwohl, 2002, p. 215). It has the subcategories generating, planning, and producing. In the first example given above, I may synthesize that: $7(10+9)=7 \quad 10+7 \quad 9$ or $(3+4) \quad 19=3 \quad 19+4 \quad 19$. Then, by performing the operations involved in the expression, I am able to find a solution for the problem. For the second example, the synthesis may be $1,-5, \_9,-13,-17 \ldots$ or $1,1+4=5,5+4=9,9+4=13$, $13+4=17 \ldots$. In contrast with the previous example, at this stage of my thinking process I am not able to make a statement about the $100^{\text {th }}$ term of the given sequence. I need to complete another mental action.

The next action included in the schemata for the generalization activity is abstraction. For the description of this process, I am using Dubinsky's review of Piaget's work, on the process of abstraction in mathematics, scattered over many articles and books written by Piaget in his last 15 years of life.

Piaget considered three stages of the process of abstraction (Dubinsky, 1991): empirical abstraction, pseudo-empirical abstraction, and reflective abstraction. First stage, the empirical abstraction, consists in deriving statements from the external properties of the given data. As Dubinsky explained, it means to extend the properties from being particular to "some" data (the given ones) to "all" possible data. For example, if I synthesize that the first terms of the sequence 1, 5, 9, 13, 17 are the odd numbers $1, \ldots 5, \ldots 9, \ldots 13, \ldots, 17$, then I can make the generalization 
that the sequence represents the odd whole numbers beginning with 1 and skips every other odd number. To find the $100^{\text {th }}$ term of the sequence, I will continue enumerating the odd numbers until I get to the $100^{\text {th }}$ term. If my synthesis for the first 5 terms of the sequence is $1,1+4=5,5+4=9,9+4=13,13+4=17$, then I can make the statement that the sequence starts with 1 and then each term is the sum from the previous term and 4 . To find the $100^{\text {th }}$ term, I will continue to add 4 to the last term of the sequence that I know until I obtain the 100 th term. In both examples, I performed an empirical abstraction because I extended an external property that exists among the 5 terms of the sequence to any of its terms.

The second level of abstraction is the pseudo-empirical abstraction. It is intermediary between empirical abstraction and reflective abstraction. The pseudoabstraction consists in deriving new properties by transforming the initial data. We obtain a new set of data after we apply some operations to the initial set of data. Let us, again, consider the case of the sequence 1, 5, 9, 13, 17... Suppose that my empirical abstraction was that the sequence is the whole odd numbers starting with 1 and skipping every other odd number. I know that each whole odd number can be written in the form $2 k+1$, where $k$ is a whole number. Therefore, I transform my initial data by rewriting each term using the previous representation. My new set of data is: $20+1=1,22+1=5,24+1=9$, $26+1=13,28+1=17, \ldots$ I make the statement that the initial sequence is the same as the sequence, $20+1,22+1,24+1,26+1,28+1, \ldots$ At this time, I performed a pseudo-empirical abstraction. If I do not know how to represent an odd number, then I will transform the initial sequence by adding the missing odd numbers, and I consider the sequence $1,3,5,7,9,11,13,15,17$, ... I analyze this new sequence, and I see that 3 is with 2 more than 1, 5 is with 2 more than 3, etc. My synthesis is that the first nine terms of the sequence are 1 , $1+2=3,3+2=5$, etc. I take out the numbers that I added to the initial sequence, and I obtain the following: $1,3+2=5,7+2=9,11+2=13,15+2=17, \ldots$ I declare now that my initial sequence is the same as $1,3+2,7+2,11+2,15+2, \ldots$ Therefore, I performed a pseudo-abstraction.

The third level of abstraction is reflective abstraction. In this process, in order to delineate general properties, the focus is on a single case and the actions became coordinated by using high mental functions that are involved in logicomathematical operations (e.g., using known mathematical laws, properties, concepts). For example, from pseudo-empirical abstraction, I have the sequence: $20+1,22+1,24+1,26+1,28+1, \ldots$ My intention is to find how I can determine the term when I know its position in the sequence. I do a new analysis, synthesis, and empirical abstraction and I declare that the terms of the sequence are of the form 2 multiplied by an even number plus 1 . I focus on the first term: $20+1$. Since, 2 and 1 are fixed in the form of a term, I coordinate my actions in order to connect 0 with the position 1. I consider the actions: 0 is an even number, $0=2 \quad 0=2 \quad\left(\begin{array}{ll}1 & 1\end{array}\right)$ and I connect the first 1 with the position of the term. Therefore, if the position of a term is $n$, then it equals $22\left(\begin{array}{ll}n & 1\end{array}\right)+1=4 \quad\left(\begin{array}{ll}n & 1\end{array}\right)+1=4 n \quad 4+1=4 n \quad 3$.

This is a general property of the initial data that was discovered through the process of reflective abstraction, as Dubinsky described it. 
The three stages of the process of abstraction interrelated. The pseudo-empirical and reflective abstractions are using the results of the empirical abstraction. Before performing a reflective abstraction the processes of empirical and pseudo-empirical abstractions may be carried out more than once. Moreover, if we consider all the actions involved in the process of generalization (analysis, synthesis, and abstraction), there is no strict order in which they are performed. However, we may direct ourselves through a generalization activity by using as reference the three routes through generalization described by Rubinshtein, which I will describe next.

\section{Rubinshtein's Three Routes Through Generalization}

As cited by Davydov (1990), Rubinshtein noticed three routes toward generalization, which describe the actions taken in order to complete the activity of generalization. The first route is called "empirical generalization". In this case, the goal of finding a general statement is attained through actions that have the purpose to determine what some given objects have in common. This action is performed through operations that lead to identify each object. These operations are usually comparisons that describe how an object is similar, or identical with other objects.

Rubinshtein described the first route is the "empirical generalization" in the following way:

“...[The elementary empirical generalization] is accomplished as a result of comparison by singling out the general (similar) properties in which the phenomena being compared coincide. ... This sort of generalization is merely a selection from a number of properties that are given empirically, directly, and sincerely; it is thus not capable of leading to the discovery of anything above what is given directly, by the senses." (Citation taken from Davydov, 1990, p. 192)

For empirical generalization, the primary mental operation is comparison, which is the method or logical technique by which the common attributes of particular objects are determined (Davydov, 1990, p. 38). The operations applied during the comparison action use only what are immediately given and the information received through senses. No transformation is performed on row data. The following example presents an empirical generalization.

Suppose that I need to find the class of the numbers 2, 4, 6, 8. First I compare 2 with 4 . I notice: that 2 and 4 are whole numbers; that they are even; that 4 is the double of 2; that 2 is half of 4 ; that 2 is the square root of 4 ; and that 4 is the square of 2 . Then, I compare 2 with 6,2 with 8 , 4 with 6,4 with 8 , and 6 with 8 . I look at all the results of my comparison, and I see that all of them are whole numbers and even (the common attributes). Therefore, I did an analysis. By synthesizing, I conclude that the numbers are even whole numbers. Now, I can 
abstract by declaring that there is an even whole numbers class that contains the given numbers. The process of generalization was successfully performed.

Unfortunately, by performing an empirical generalization we cannot guaranty the success in all situations, as I will illustrate with the next example. Suppose that I need to find the next term in the sequence $1,5,9,13,17 \ldots$. First I compare 1 with 5. I notice that 1 and 5 are whole numbers, that they are odd. Then, I compare 1 with 9,1 with 13,1 with 17,5 with 9,5 with 13,5 with 17,9 with 13,9 with 17, and 13 with 17. I look at all the results of my comparison, and I notice that all of them are whole numbers, and they are odd (the common attributes). Therefore, I did an analysis. By synthesizing, I conclude that the numbers are odd whole numbers. Now, I can that there is an odd whole numbers class that contains the given numbers, which is true but the process of generalization is not finished. If I say that the general term is $2 n+1$, the general representation of an odd number, I do not give the correct answer since $2 x+1=3$ is not a member of the sequence. Therefore, I did not perform a successful generalization. At this point, we need to look for a different approach to solve the problem.

The second route to generalization described by Rubinshtein (as presented by Davydov, 1990) is to focus the mental activity on analysis and abstraction. The purpose of the analysis is to distinguish what is essential from what is not essential. The essential of an object is a characteristic that remains unchanged in the object when it is transformed during its interactions with other objects. When the essential is delineated, it becomes right away abstracted. Then the abstract can be synthesized into a concrete conclusion, by a mental restoration and interpretation of the observed phenomena. This generalization is called scientific or theoretical generalization and it is described as:

"Not merely a selection but a transformation as well... The transformation of what is immediately given, which leads to an abstract concept of a phenomenon, consists in breaking the contact ... of the attendant circumstances, which complicate or mask the essence of phenomena." (Rubinshtein, cited by Davydov, 1990, p. 193)

I will use the second route to generalization to the previous example where the problem was to find the next term in the sequence 1, 5, 9, 13, 17... I start looking at each number, and I observe that each of them is odd. My next step is to analyze what remains unchanged in a number when it interacts with the numbers that are close to it. I pick up an object, for example, 5 (I would not pick 1 because it has fewer interactions with other objects). I see that 5-1=4 and that 9 $5=4$ (I transformed the give data). Analyzing the results of this transformation, I see that the distance between 5 and its neighbors is the same, 4 . I make the abstraction that each term of the sequence is with 4 greater than its left neighbor and with 4 less than its right neighbor. I synthesize that 9 is with 4 less than 13, and that 13 is with 4 less than 17. I do another analysis, and I see that the relation between a term and its predecessor is always the same, 4. Now, I make the abstraction that every term is its predecessor plus 4 . Finally, I say that the next term in the sequence is $17+4=21$. Therefore, by following the second route to 
generalization described by Rubinstein I was able to generalize the relationship between the numbers and I applied this general relation to solve the problem. The question is, now, what should I do if the problem would seek to find the general term of the sequence $1,5,9,13,17 \ldots$ ?

Rubinshtein mentioned a third route through generalization that can be taken. This route requires a theoretical derivation that is "accomplished by a two-way movement from general to the particular and from the particular to the general generalization and theoretical cognition are interrelated" (Davydov, 1990, p. 194).

In the case of the problem that requests the general term for the sequence 1, 5, 9, $13,17 . .$. I will start from the general statement that I made before: each term is its predecessor+4. I will use symbols to present this statement in the following way: $a_{n+1}=a_{n}+4$. I will apply this general formula to the first particular cases:

$a_{2}=a_{1}+4=1+4, a_{3}=a_{2}+4=1+4+4$ and so on.

By analyzing the transformations that I performed, I notice that for each term has 1 as a constant component and that 4 is a repeated addend. I do the abstraction that $a_{n}=1+\left(\begin{array}{ll}n & 1\end{array}\right) \times 4$. I am checking now if the formula is working for the first terms of the sequence. The proposition $5=1+\left(\begin{array}{ll}2 & 1\end{array}\right) \times 4$ is true. Also, the proposition $9=1+(31) \times 4$ is true. For this special case in which the problem involves a number sequence, to prove that the abstract formula represents the general term, I will show that if $a_{k}=1+\left(\begin{array}{ll}k & 1\end{array}\right) \times 4$ is true, then $a_{k+1}=1+k \times 4$ is true. That is: $a_{k+1}=a_{k}+4=1+\left(\begin{array}{ll}k & 1\end{array}\right) \times 4+4=1+k \times 4 \quad 4+4=1+k \times 4$ Therefore, $a_{k+1}=1+k \times 4$ is true.

In the later computations, I used the letter $k$ instead of $n$ because I wanted to emphasize that those computations are general but preformed for the particular situation where the formula for $a_{k+1}$ can be derived from the formula of $a_{k}$. This is an instance in which we see the two-way move from general to particular and from particular to general. Now, I decide that the general term for the given sequence is $a_{n}=1+\left(\begin{array}{ll}n & 1\end{array}\right) \times 4$. The technique that I used to determine the general formula is known in mathematics as mathematical induction. This method is a relevant example for the third route to generalization described by Rubinstein.

\section{Three Categories of Mathematical Generalization Activities}

Similar to Rubinshtein's description of the three types of thinking processes that may develop during a generalization activity, in mathematical activities we may consider three routes that lead toward generalization: the empirical generalization, the pseudo-empirical generalization, and the reflective generalization. The key element, which differentiates the generalization activities, is the purpose of the action of abstraction. Therefore, the categories of mathematical generalization activities follow the three "routes" through generalization described by Rubinshtein empowered by the categories of abstraction resumed by Dubinsky from Piaget's work. 
An empirical generalization activity involves the actions of analysis, synthesis, and empirical abstraction. The purpose of the action of analysis is to separate the problem situation into distinct parts. Then, by comparing the parts among them, we determine the common properties of these components. Suppose that we need to find the area of a triangle that has the length of the base $7 \mathrm{~cm}$ and a height of $4 \mathrm{~cm}$. By analyzing this statement, we can separate the following parts: a triangle with a base of $7 \mathrm{~cm}$ long and a height of $4 \mathrm{~cm}$ long; and the concept of area of a triangle. Both parts have in common the geometrical shape of a triangle. We may synthesize this information. This means that we may describe the situation by using the observations made during the action of analysis. Therefore, we have a triangle (base $=7 \mathrm{~cm}$, height $=4 \mathrm{~cm}$ ) that has an area. The area of a triangle can be computed by using the general formula Area $_{\text {triangle }}=\frac{\text { base height }}{2}$. Now, we can find the area of the given triangle: Area $=\frac{7 \mathrm{~cm} 4 \mathrm{~cm}}{2}=14 \mathrm{~cm}^{2}$. The final result was the outcome of an empirical abstraction. We used only external properties of the specified triangle and the general formula for the area of a triangle.

The second category of mathematical generalization activities involves the pseudo-empirical generalization. The actions used in this process are analysis, synthesis, and pseudo-abstraction. Suppose that we need to determine the area of a right triangle that has the legs of lengths $7 \mathrm{~cm}$ respective $4 \mathrm{~cm}$. The analysis and synthesis actions are similar to the previous example, but we cannot go further with an empirical abstraction because the external properties of the parts of the problem do not match. The given triangle is a right triangle with its legs of $7 \mathrm{~cm}$ and $4 \mathrm{~cm}$. The second part is a general formula for the area of a triangle that uses the lengths of the base and height. We have to return to the information that we have from the action of analysis. We have a right triangle, and we need to find the lengths of its base and height in order to compute its area. We know that in a triangle we can choose as a base any of its side. Therefore, if in the right triangle we choose the leg of side $4 \mathrm{~cm}$ as the base, then the leg of side $7 \mathrm{~cm}$ becomes the height of the triangle. With this transformation of the initial information, we are able to compute the area that we need to find. We obtained the solution through a pseudo-empirical abstraction.

The third category of mathematical activities is the reflective generalization. In a reflective generalization, the actions involved are analysis, synthesis and reflective abstraction. Assume that we need to find the area of an equilateral triangle with the side of length $7 \mathrm{~cm}$. The information that we have from analysis and synthesis is that we have an equilateral triangle of side $7 \mathrm{~cm}$ and we have to know the lengths of its base and height. Through pseudo-empirical abstraction, we transform the initial data by considering the length of the base of the triangle equal with $7 \mathrm{~cm}$. In the information, that we have, there are no data about the height of the triangle. We have to do a reflective abstraction in order to find the length of the triangle's height. 
Therefore, we start construct an equilateral triangle in which I draw its height. We know that the height of the equilateral triangle divides the base in half (if we do not know this property, we have to do another reflective generalization activity). We separate from the first equilateral triangle a half that forms a right triangle with one of the legs being the height of the initial triangle, the second leg is a half of the base of the initial triangle (its length is $\frac{7}{2} \mathrm{~cm}$ ), and the hypotenuse is a side of the first triangle (its length is $7 \mathrm{~cm}$ ). In the new triangle, we use a consequence of the Pythagorean Theorem. Therefore, we have that $L e g=\sqrt{(7 \mathrm{~cm})^{2} \quad \frac{7}{2} \mathrm{~cm} \div}=\frac{7 \sqrt{3}}{2} \mathrm{~cm}$. Now, we reverse the reasoning process. We have the length of the leg of the right triangle, which was separated from the initial equilateral triangle. We reconstruct the equilateral triangle using the new information that we have. Now, we have a triangle with a base of length $7 \mathrm{~cm}$ and a height of $\frac{7 \sqrt{3}}{2} \mathrm{~cm}$. The area of the equilateral triangle of side $7 \mathrm{~cm}$ is Area $=\frac{7 \mathrm{~cm} \frac{7 \sqrt{3}}{2} \mathrm{~cm}}{2}=\frac{49 \sqrt{3}}{4} \mathrm{~cm}^{2}$.

The examples, which I used to illustrate the three categories of mathematical generalization activities, are all focused to identify something general that is already known (the general formula for the area of a triangle) and apply it in particular cases. For situations when we need to find something general that is not known from isolated and particular cases, the examples used to illustrate Rubinshtein's three routes through generalizations are reasonable representations for the three categories of mathematical generalization activities.

\section{Conclusion}

To conclude, the generalization process in mathematics can be regarded as an activity system. By explaining the means of the generalization activity using Leontief's activity theory and Rubinshtein's descriptions of the generalization process, we are able to follow the thinking trends that are behind a mathematical generalization. The understanding of the process of generalization in mathematics as an activity system is only the first step in developing a perspective of teaching mathematics through generalization. This paper is aimed to provide a space for reflection on what it means to perform a generalization in mathematics and a starting point for a new work approach in several areas of research: student learning, teacher education, curriculum development.

In the realm of student learning, the theory may be used to get more insight in students' development of mathematics reasoning and to identify the correlation between the biological development and the process of generalization in mathematics. For the area of teacher preparation and teacher practices research, the theory may be used to identify the support needed for implementing high leverage teaching practices (TeachingWorks, 2017) into school curriculum. Some research questions that arise are: What questions do teachers ask in order to 
provoke student's sharing about their analysis, synthesis, and their abstraction level? What patterns of abstraction, synthesis, and abstraction do students use while they work on a mathematical task? How do teachers lead a classroom discussion in order to elevate students' abstract thinking? In the area of curriculum development, we have to develop and research designing curricula and classroom activities that would follow the principles of generalization.

There are a couple of limitations for the way in which I interpret the activity of generalization. First, Leont'ev strongly highlighted the "essential" connection between the motive for an activity and the activity itself, which will not be too much emphasized in my description. This aspect of an activity involves a separate analysis from the position of motivational theory. My focus is on what is happening after the motivational level. The second limitation, which is the most critical, is that the definition is purely theoretical. However, it is a valuable tool to analyze classroom activities.

\section{References}

Davydov, V. V. (2008). Problems of developmental instruction: a theoretical and experimental psychological study. Nova Science, NY.

Davydov, V. V. (1990). Types of generalization in instruction: Logical and psychological problems in the structuring of school curricula (Soviet Studies in Mathematics Education, Vol. 2). Reston, VA: National Council of Teachers of Mathematics.

Dougherty, B. \& Slovin, H. (2004). Generalised diagrams as a tool for young children's problem solving. In M. J. Høines \& A. B. Fuglestad (Eds.), Proceedings of the 28th annual conference of the International Group for the Psychology of Mathematics Education (Vol. 2, pp. 295-302). Bergen, Norway: PME.

Dubinsky E. (1991) Constructive Aspects of Reflective Abstraction in Advanced Mathematics. In: Steffe L.P. (eds) Epistemological Foundations of Mathematical Experience. Recent Research in Psychology. Springer, New York, NY. https://doi.org/10.1007/978-1-4612-3178-3_9

Engestrom, Y., (2001) Expansive Learning at Work: Toward an activity theoretical reconceptualization, Journal of Education and Work, 14(1), 133-156. https://doi.org/10.1080/13639080020028747

Gedera, D. S. P. \& Williams, D. S. P. (Eds.). (2016). Activity Theory in Education: Research and Practice. SensePublishers, Rotterdam. https://doi.org/10.1007/978-94-6300$387-2$

Harry, D., (2008). Reflections on points of departure in the development of sociocultural and activity theory. In Bert van Oers, Wim Wardekker, Ed Elbers, René van der Veer (Eds.). The transformation of learning : advances in cultural-historical activity theory. Cambridge University Press: Cambridge ; New York. https://doi.org/10.1017/cbo9780511499937.006

Jooganah, K., Williams, J. S., (2016). Contradictions between and within school and university activity systems helping to explain students' difficulty with advanced mathematics. Teaching Mathematics and its Applications: An International Journal of the IMA, 35(3),159-171. https:// doi.org/10.1093/teamat/hrw014

Kaput, J. J., Carraher, D. W., \& Blanton, M. L. (Eds.). (2008). Algebra in the Early Grades. New York: Routledge, Taylor \& Francis Group. https://doi.org/10.4324/9781315097435

Krathwohl, D.R. (2002). A revision of Bloom's taxonomy: an overview. Theory Into Practice, 41(4), 212-218. https://doi.org/10.1207/s15430421tip4104_2

Krutetskii,V . A. (1976). The psychology of mathematical abilities in schoolchildren, (translated from the Russian by J. Teller). Chicago,IL: University of Chicago Press. 
https:// doi.org/10.2307/748528

Lee, L., (1996). An initiation into algebraic culture through generalization activities. In N. Bednarz, C. Kieran, and L. Lee, (Eds.). Approaches to Algebra: Perspectives for Research and Teaching, Kluwer, Dordrecht/Boston/London, pp. 87-106. https://doi.org/10.1007/978-94-009-1732-3_6

Leontiev, A. (1978). Activity, Consciousness, and Personality. Englewood Cliffs, NJ: Prentice-Hall.

Mason, J. (1996). Expressing generality and roots of algebra. In N. Bednarz, C. Kieran, and L. Lee, (Eds.). Approaches to Algebra: Perspectives for Research and Teaching, Kluwer, Dordrecht/Boston/London, pp. 65-86. https://doi.org/10.1007/978-94009-1732-3_5

Moxhay, P. (2008). Assessing the scientific concept of number in primary school children. Paper presented at ISCAR 2008, San Diego.

Piaget, J. (1964). Development and learning. In: R.E. Ripple and V.N. Rockcastle, Editors. Piaget rediscovered. Cornell University Press, Ithaca, 7-19. https://doi.org/10.1002/tea.3660020305

Rubinshtein, S. L. (1994). Thinking and ways of investigating it. Journal Of Russian and East European Psychology, 32 (5), 63-93. https://doi.org/10.2753/rpo1061-0405320563

Schmittau, J. (2003). Cultural-historical theory and mathematical education. In

Vygotsky's educational theory in cultural context. NY: Cambridge University Press. https://doi.org/10.1017/cbo9780511840975.013

Schmittau, J. (2004). Vygotskian theory and mathematics education: Resolving the conceptual-procedural dichotomy. European Journal of Psychology of Education, XIX, 19-43. https://doi.org/10.1007/bf03173235

TeachingWorks (2017). TeachingWorks: High-leverage practices. University of Michigan. Retrieved from: http://www.teachingworks.org/work-of-teaching/highleverage-practices.

Vygotsky, L. S. (1978). Mind in society: The development of higher psychological Processes. M. Cole, V. John-Steiner, S. Scribner, \& E. Souberman (Eds.). Cambridge, MA: Harvard University Press. https://doi.org/10.2307/1421493

Vygotsky, L. S. (1986). Thought and language. A. Kozulin (Ed). Cambridge, MA: MIT Press. https://doi.org/10.1017/S0272263100008172 